\title{
Modeling the Long-Term Cost-Effectiveness of IDegLira in Patients with Type 2 Diabetes Who are Failing To Meet Glycemic Targets on Basal Insulin Alone in The Netherlands
}

\author{
Barnaby Hunt · Divina Glah · Maarten van der Vliet
}

Received: April 5, 2017 / Published online: May 18, 2017

(C) The Author(s) 2017. This article is an open access publication

\begin{abstract}
Introduction: Insulin degludec/liraglutide (IDegLira) is the first basal insulin and glucagon-like peptide-1 receptor agonist (GLP-1 RA) in a single pen injection device, and a oncedaily treatment option for patients with type 2 diabetes mellitus (T2DM) who are uncontrolled on basal insulin and require treatment intensification. The objective of this analysis was to evaluate the long-term cost-effectiveness of IDegLira versus basal-bolus therapy (insulin glargine $\mathrm{U} 100+3 \times$ daily insulin aspart) for patients with T2DM uncontrolled on basal insulin $\left[\mathrm{HbA}_{1 \mathrm{c}}>53 \mathrm{mmol} / \mathrm{mol}(>7 \%)\right]$ in the Netherlands.
\end{abstract}

Enhanced content To view enhanced content for this article go to http://www.medengine.com/Redeem/ 3848F0600D95B0D2.

Electronic supplementary material The online version of this article (doi:10.1007/s13300-017-0266-3) contains supplementary material, which is available to authorized users.

\section{B. Hunt}

Ossian Health Economics and Communications, Bäumleingasse 20, 4051 Basel, Switzerland

D. Glah

Novo Nordisk Ltd, Gatwick, West Sussex, UK

M. van der Vliet $(\bowtie)$

Novo Nordisk BV, Alphen aan den Rijn,

The Netherlands

e-mail: mtvt@novonordisk.com
Methods: Cost-effectiveness analysis was performed using the validated IMS CORE Diabetes Model from a healthcare payer perspective. Outcomes were modeled over patient lifetimes in a cohort with baseline characteristics from the DUAL ${ }^{\mathrm{TM}}$ II trial. Treatment effect data were sourced from a statistical indirect comparison (pooled analysis) of IDegLira with basal-bolus therapy.

Results: Treatment with IDegLira resulted in mean increases in quality-adjusted life expectancy of 0.43 quality-adjusted life years versus basal-bolus therapy. Improved clinical outcomes resulted from fewer diabetes-related complications and a delayed time to their onset. IDegLira was associated with lower costs of EUR 4679 versus basal-bolus therapy, a result of lower pharmacy costs and avoided diabetes-related complications. Thus, IDegLira was dominant, i.e., both more effective and less costly than basal-bolus therapy.

Conclusions: IDegLira is an effective treatment option to improve glycemic control without incurring an increased risk of hypoglycemia or weight gain. This analysis suggests that IDegLira is cost-effective versus basal-bolus therapy in patients with T2DM who are uncontrolled on basal insulin in the Netherlands.

Funding: Novo Nordisk.

Keywords: GLP-1 analogs; $\mathrm{HbA}_{1 \mathrm{c}}$; Hypoglycemia; Insulin analogs; Type 2 diabetes 


\section{INTRODUCTION}

Diabetes mellitus is a chronic metabolic disorder characterized by elevated levels of blood glucose (hyperglycemia). The disease is associated with considerable morbidity and mortality [1] and is one of the biggest health challenges facing the world. The International Diabetes Federation (IDF) estimated that, in 2015, almost one million people in the 20-79 year age group in the Netherlands had diabetes $(7.9 \%$ of the population) and there were approximately 7500 diabetes-related deaths [2]. Healthcare spending in the Netherlands as a result of diabetes mellitus and its related complications was estimated to be around EUR 6.2 billion in 2015 [2].

The clinical goal of the treatment of diabetes is to achieve good glycemic control with minimal hypoglycemia and other adverse effects of treatment. In the Netherlands, guidance for the management of hyperglycemia in type 2 diabetes mellitus (T2DM) recommends a patient-centered approach that considers age, intensity of treatment, and diabetes duration, with a general $\mathrm{HbA}_{1 \mathrm{c}}$ target of $\leq 53 \mathrm{mmol} / \mathrm{mol}$ (7\%) [3].

Over the past five years, annual evaluations of Dutch diabetes care programs offered in primary care have consistently shown that around $65-70 \%$ of patients aged below 70 years are on target in terms of $\mathrm{HbA}_{1 \mathrm{c}}$ [4]. However, a recent database study of patients with T2DM in the Netherlands found that a substantial proportion of basal-only insulin users (73\%) did not achieve their glycemic control targets $\left(\mathrm{HbA}_{1 \mathrm{c}} \leq 53 \mathrm{mmol} / \mathrm{mol} ; \leq 7 \%\right)$, and only about one-third underwent intensification of basal insulin therapy during a median follow-up of 14 months [5]. In the Netherlands, the top three most commonly cited barriers to insulin intensification include multiple daily injections, the complexity involved in calculating the correct bolus dose, and weight gain [6].

Once-daily IDegLira is a combination of a long-acting basal insulin analog (insulin degludec; IDeg) and a GLP-1 receptor agonist (liraglutide) administered in a single pen injection device. IDegLira is indicated for the treatment of adults with T2DM to improve glycemic control in combination with oral glucose-lowering medicinal products when these alone or combined with a GLP-1 RA or basal insulin do not provide adequate glycemic control. A suggested place in the T2DM treatment pathway for IDegLira is when patients are uncontrolled on basal insulin and require treatment intensification.

Core efficacy and safety evidence for IDegLira in patients with T2DM uncontrolled on basal insulin is provided by two phase- 3 DUAL $^{\text {TM }}$ (Dual Action of Liraglutide and Insulin Degludec in Type 2 Diabetes) trials, DUAL II (NCT 01392573) [7] and DUAL V (NCT01952145) [8]. DUAL II compared IDegLira with IDeg and found that IDegLira was superior to IDeg at equivalent insulin doses in terms of lowering $\mathrm{HbA}_{1 \mathrm{c}}$, confirmed that hypoglycemia was numerically lower, and found that the change in body weight was significantly more favorable with IDegLira (weight loss) versus IDeg (no weight change) [7]. DUAL $V$ investigated the efficacy of IDegLira versus uptitration of insulin glargine U100 (IGlar U100; Lantus ${ }^{\circledR}$ ) in patients with T2DM uncontrolled on IGlar U100 at trial entry. IDegLira was superior to IGlar U100 in terms of lowering $\mathrm{HbA}_{1 \mathrm{c}}$, change in body weight (weight loss with IDegLira versus weight gain with IGlar U100), and hypoglycemia. Despite a superior reduction in $\mathrm{HbA}_{1 \mathrm{c}}$, the rate of confirmed hypoglycemia was $57 \%$ lower with IDegLira [8].

There are currently no direct head-to-head clinical trials of IDegLira versus other treatment options for intensification of basal insulin therapy, such as basal-bolus therapy or GLP-1 RA added to basal insulin. A statistical indirect comparison (pooled analysis) has been conducted to establish an estimate of the treatment effects of IDegLira versus these treatment regimens in patients with T2DM uncontrolled on basal insulin; the results of the pooled analysis have been published elsewhere [9]. The pooled analysis shows that IDegLira achieves a significantly greater decrease in $\mathrm{HbA}_{1 \mathrm{c}}$ along with lower hypoglycemia rates than basal-bolus therapy, as well as significant improvements in body weight (weight loss with IDegLira versus weight gain with basal-bolus therapy) [9]. 
In order to optimize resource use and service delivery for patients with T2DM, decision-making based on both clinical and economic evidence is essential. Cost-effectiveness analyses are increasingly used to inform pharmaceutical reimbursement and/or pricing decisions in many countries. The objective of the present study was to investigate the cost-effectiveness of IDegLira versus the alternative option of basal-bolus therapy for intensification of basal insulin therapy in patients with T2DM uncontrolled on basal insulin in the Netherlands.

\section{METHODS}

\section{Choice of Comparator}

According to national guidelines in the Netherlands, patients with T2DM who are failing on basal insulin should progress to basal-bolus therapy or premix insulin. Based on internal calculations of IMS health databases, anatomical therapeutic chemical class 4-moving annual total Q2 2016, the majority of patients within the Netherlands are switched to basal-bolus therapy. The same data show that human insulin accounts for only 5.8\% of the total insulin sold. Therefore, the most relevant comparator for IDegLira in the Netherlands was considered to be the addition of three-times-daily insulin aspart to once-daily insulin analog IGlar U100 (IGlar U100 OD + 3× IAsp).

\section{Model overview}

A cost-utility analysis was used to compare IDegLira with IGlar U100 (Lantus $^{\circledR}$ ) $+3 \times$ IAsp (basal-bolus therapy) in patients with T2DM uncontrolled on basal insulin. The main outcome measure was the incremental cost-effectiveness ratio (ICER) in terms of cost per quality-adjusted life year [QALY] gained) [10]. The ICER allows comparison of the value of alternative treatment options for a specific therapeutic indication and is the preferred outcome measure of many health technology assessment bodies. No formal ICER threshold value has been defined so far in the Netherlands [11]. A cutoff value of EUR 20,000 per QALY gained is sometimes discussed, and the Council for Care and Public Health suggests an absolute maximum ICER threshold value of EUR 80,000 per QALY gained, but other factors play a role in the decision-making process [11].

Long-term clinical and economic outcomes were estimated using the IMS CORE Diabetes Model, an internet-based interactive computer model developed to determine the long-term health outcomes and economic consequences of implementing interventions in the treatment of diabetes $[12,13]$. The architecture, assumptions, features, and capabilities of the model have been published previously [13]. The model is a validated, non-product-specific diabetes policy analysis tool that allows extrapolation of results from short-term trials to long-term outcomes. It accounts for diabetes therapy, oral hypoglycemic medications, screening and treatment strategies for microvascular complications, treatment strategies for end-stage complications, and multifactorial interventions.

Costs were estimated from a healthcare payer perspective in the Netherlands. All costs were expressed in 2015 EUR. Clinical outcomes captured all direct health effects on the patient. Indirect costs were not included in the present analysis, as the required Netherlands-specific days off work estimates for each diabetes-related complication could not be identified. This is likely to be a conservative approach, as IDegLira is associated with a reduced incidence of complications and therefore less lost productivity. Clinical outcomes were discounted at $1.5 \%$ per annum and cost outcomes were discounted at $4 \%$ per annum, in line with health economic guidance for the Netherlands [14].

\section{Compliance with Ethics Guidelines}

This article is based on previously conducted studies, and does not involve any new studies of human or animal subjects performed by any other authors.

\section{Time Horizon and Treatment Duration}

The base case analysis used a lifetime (50-year) time horizon to capture all relevant long-term 
complications and associated costs in order to assess their impact on life expectancy and quality-adjusted life expectancy. The model takes into account mortality as a result of diabetes-related complications and background mortality based on Netherlands-specific life tables [15]. Therefore, whilst a 50-year time horizon was used, patients were not assumed to live for 50 years. All patients had died after 50 years of the modeling analysis.

Patients receiving IDegLira were assumed to receive that treatment for the first five years of the analysis, after which they were switched to basal-bolus therapy (IGlar U100 OD $+3 \times$ IAsp). This assumption recognizes that intensification to basal-bolus therapy will likely be required for patients to maintain glycemic control over the long-term. Each patient already receiving basal-bolus therapy was assumed to remain on it for the duration of their lifetime.

\section{Clinical Data}

A simulated cohort of patients was defined, with baseline risk factors based on the baseline characteristics of patients randomly allocated to receive IDegLira in the DUAL II study; see Table 1. The proportion of patients using tobacco products was based on the trial data, but the number of cigarettes smoked per day was assumed to be the same as the general Netherlands population and was based on country-specific data [16]. Similarly, mean weekly alcohol consumption was taken from Netherlands-specific data for the general population [17].

Treatment effects for IDegLira and basal-bolus therapy applied in the first year of the analysis (Table 2) were based on data from the pooled analysis [9]. After the first year, systolic blood pressure and serum lipids were assumed to follow the natural progression algorithms built into the IMS CORE Diabetes Model, based on the UK Prospective Diabetes Study (UKPDS) or Framingham data (as described by Palmer et al. [13]). Benefits in terms of $\mathrm{HbA}_{1 \mathrm{c}}$ were assumed to persist for the 5 years that patients received IDegLira and were abolished on switching treatment. The BMI benefit was also
Table 1 Baseline cohort characteristics

\begin{tabular}{ll}
\hline Characteristic & $\begin{array}{l}\text { DUAL II c cohort } \\
\text { (patients receiving } \\
\text { IDegLira) }\end{array}$ \\
\hline Demographics and risk factors, mean & $(\mathrm{SD})$ \\
Start age (years) & $56.8(8.9)$ \\
Duration of diabetes (years) & $10.3(6.0)$ \\
Percentage male (\%) & 56.3 \\
HbA ${ }_{1 \mathrm{c}}(\%)$ & $8.7(0.7)$ \\
SBP (mmHg) & $132.4(14.8)$ \\
Total cholesterol (mg/dL) & $182.0(45.5)$ \\
HDL cholesterol (mg/dL) & $43.4(11.0)$ \\
LDL cholesterol (mg/dL) & $101.9(37.1)$ \\
Triglycerides (mg/dL) & $196.8(148.0)$ \\
BMI (kg/m $\left.{ }^{2}\right)$ & $33.6(5.7)$ \\
Percentage smokers (\%) & 16.1 \\
Cigarettes per day & \\
Alcohol consumption & 12.7 \\
(fl oz/week) & 4.66 \\
Ethnic group, \% & \\
White & \\
Black & \\
Hispanic & 70.9 \\
Native American & 4.5 \\
Asian/Pacific Islander & 0 \\
\hline
\end{tabular}

$B M I$ body mass index, $H b A_{I c}$ glycated hemoglobin, $H D L$ high-density lipoprotein, $L D L$ low-density lipoprotein, SBP systolic blood pressure

${ }^{a}$ From [16]

b From [17]

c DUAL II is a randomized, controlled, double-blind, multinational, treat-to-target trial in which IDegLira was compared with IDeg over 26 weeks of treatment in patients with T2DM uncontrolled on basal insulin [7]

assumed to persist whilst patients remained on IDegLira and was abolished on treatment intensification. On intensification, an increase was applied in the IDegLira arm to abolish the 
Table 2 Treatment effects applied in patients previously uncontrolled on basal insulin Source: supplementary appendix in [9] with pooled analysis. The basal-bolus arm of the pooled analysis that was used to inform this analysis included patients who previously received IGlar $\mathrm{U} 100+3 \times$ IAsp and IDeg $+3 \times$ IAsp. Whilst pooled treatment effects were used to explore changes in physiological parameters, unit costs of IGlar U100 were used to calculate annual treatment costs. This is likely to be a conservative approach, since IDeg is associated with lower rates of hypoglycemia and reduced weight gain compared with IGlar U100 but IGlar U100 is associated with a lower cost

\begin{tabular}{lll}
\hline Parameter [mean $(\mathrm{SD})]$ & IDegLira $(\boldsymbol{n}=\mathbf{1 9 9})$ & $\begin{array}{l}\text { IGlar U100 + 3× IAsp } \\
(\boldsymbol{n}=\mathbf{2 1 0})\end{array}$ \\
\hline $\mathrm{HbA}_{1 \mathrm{c}}(\%)$ & $-1.66(0.96)$ & $-1.33(0.96)^{*}$ \\
$\mathrm{SBP}(\mathrm{mmHg})$ & $-6.86(13.20)$ & $-0.93(13.20)^{*}$ \\
Total cholesterol $(\mathrm{mg} / \mathrm{dL})$ & $-10.13(30.28)$ & $+1.50(30.28)^{*}$ \\
$\mathrm{HDL}$ cholesterol $(\mathrm{mg} / \mathrm{dL})$ & $+0.52(6.79)$ & $+0.79(6.79)$ \\
$\mathrm{LDL}$ cholesterol $(\mathrm{mg} / \mathrm{dL})$ & $-6.85(23.83)$ & $+0.08(23.83)^{*}$ \\
Triglycerides $(\mathrm{mg} / \mathrm{dL})$ & $-25.74(103.71)$ & $+3.82(103.71)^{*}$ \\
$\mathrm{BMI}\left(\mathrm{kg} / \mathrm{m}^{2}\right)$ & $-1.04(1.34)$ & $+1.38(1.34)^{*}$ \\
Severe hypoglycemia event rate (events/100 PYE) (95\% CI) & 0.84 & 2.85 \\
Nonsevere hypoglycemia event rate (events/100 PYE) (95\% CI) & 125.05 & $794.63 *$ \\
Actual daily basal insulin (U) at EOT & $37.27(30.22)$ & $68.22(30.22)^{*}$ \\
Actual daily bolus insulin (U) at EOT & - & $57.88(\mathrm{NR})$ \\
\hline
\end{tabular}

$B M I$ body mass index, $C I$ confidence interval, $E O T$ end-of-trial, $H b A_{1 c}$ glycated hemoglobin, $H D L$ high-density lipoprotein, $L D L$ low-density lipoprotein, $N R$ not reported, $O D$ once daily, $P Y E$ patient years of exposure, $S D$ standard deviation, $S B P$ systolic blood pressure

* Statistically significant difference between treatment arms

difference, with no change applied in the IGlar $\mathrm{U} 100+3 \times$ IAsp arm of the analysis. Hypoglycemia rates following treatment intensification were also based on the basal-bolus arm. When patients intensified to receive IGlar $\mathrm{U} 100+3 \times$ IAsp after 5 years, nonsevere and severe hypoglycemic event rates of 794.63 and 2.85 events per 100 patient years, respectively, were applied.

\section{Costs and Resource Use}

Costs were estimated from a Netherlands healthcare payer perspective. Direct costs captured included pharmacy costs, costs associated with diabetes-related complications, and concomitant patient management costs. Treatment costs are correct as of April 2016. All other costs are expressed in 2015 EUR (inflated to 2015 EUR based on the consumer prices index where relevant).
Treatment costs were based on the clinical data from which the treatment effects were taken, with doses adjusted as part of the pooled analysis [9]. One needle was assumed for each injection. Patients were assumed to be receiving metformin in addition to the study medication. Following treatment intensification to basal-bolus therapy, treatment costs were the same in both arms (matched to IGlar $\mathrm{U} 100+3 \times$ IAsp). Patients receiving IDegLira were assumed to use one self-monitored blood glucose (SMBG) test per day (comprising one SMBG test strip and one lancet), and patients receiving IGlar U100 $+3 \times$ IAsp were assumed to use four SMBG tests per day.

Resource use relating to patient management was assumed to be the same as the general population with T2DM in the Netherlands in both treatment arms. Patient management costs captured in the analysis included concomitant 
Table 3 Sensitivity analyses conducted

Time horizon

Statistically significant differences only

$\mathrm{HbA}_{1 \mathrm{c}}$ progression

Upper and lower limits of $\mathrm{HbA}_{1 \mathrm{c}}$ change

BMI progression

Treatment switching patterns

Application of alternative insulin costs

Alternative dosing in the comparator arm

Alternative SMBG costs

Alternative needle costs
An alternative time horizon of 30 years was investigated. A time horizon of 50 years was required for all modeled patients to have died, and therefore shorter time horizons do not capture all complications and costs

Only the treatment effects that were significantly different between the IDegLira and IGlar U100 + 3× IAsp arms were applied

Two alternative approaches to $\mathrm{HbA}_{1 \mathrm{c}}$ progression were explored. In the first, the UKPDS $\mathrm{HbA}_{1 \mathrm{c}}$ progression equation was applied in both arms of the simulation. $\mathrm{HbA}_{1 \mathrm{c}}$ increased over time in both arms of the analysis, with the $\mathrm{HbA}_{1 \mathrm{c}}$ benefit in the IDegLira arm gradually reducing. In the second, no $\mathrm{HbA}_{1 \mathrm{c}}$ changes were applied following the treatment effects applied in the first year of the analysis. This attempts to capture the legacy effect, where an early improvement in $\mathrm{HbA}_{1 \mathrm{c}}$ has a benefit in the later years of life, even if the $\mathrm{HbA}_{1 \mathrm{c}}$ difference no longer persists

Simulations were run with the upper and lower 95\% confidence interval of the modeled $\mathrm{HbA}_{1 \mathrm{c}}$ change seen in the IDegLira arm

The base case analysis assumed that the BMI benefit associated with IDegLira was abolished on treatment switching. In this analysis, $50 \%$ of the benefit with IDegLira over IGlar U100 + 3× IAsp was maintained over the duration of the analysis

Simulations were performed with the year of treatment switch to basal-bolus therapy in the IDegLira arm brought forward to the end of year 3 or pushed back to the end of year 7 or year 10

As national guidelines recommend that patients with T2DM commence NPH insulin treatment before progressing to insulin analogs, the cost of IGlar U100 was replaced with the cost of NPH insulin. NPH insulin is associated with a lower cost but an increased rate of hypoglycemia compared with IGlar U100. Conservatively, no changes to the clinical inputs were applied. In another analysis, the pack price of IGlar U100 was reduced by $20 \%$

Three scenarios with alternative dosing were evaluated. (1) The observed trial doses (IDegLira: 44.8 dose steps; IGlar U100 + 3× IAsp: 71.7 IU IGlar U100 and 66.3 IU IAsp) were used to calculate annual treatment costs. (2) The maximum dose of IDegLira was used in the 5 years that patients received IDegLira. (3) Doses received in clinical practice in the Netherlands were used (44.0 IU IGlar U100 and 36.0 IU IAsp, with the dose of IDegLira unchanged from the base case analysis)

Alternative costs of SMBG test strips were investigated. One scenario applied the cost of the lowest-priced SMBG test available in the Netherlands (EUR 0.1442 per test strip). The second scenario used the average cost of a SMBG test strip in the Netherlands (EUR 0.8346 per test strip). Changes were applied in both treatment arms

Alternative needle costs were applied in both treatment arms. The cost of the lowest-price needle available in the Netherlands (EUR 0.135) was applied for all needles, and the average needle cost in the Netherlands (EUR 0.1992 per needle) was applied for all needles 
Table 3 continued

Costs of complications

Hypoglycemia disutilities

Probabilistic sensitivity analysis

The cost of treating complications was increased by $20 \%$ and reduced by $20 \%$

The effect of applying alternative disutilities for severe and nonsevere events was assessed by using the values published by Currie et al. ( -0.0118 per severe hypoglycemic event and -0.0035 per nonsevere hypoglycemic event)

PSA was conducted in the IMS CORE Diabetes Model. Continuous input parameters and regression coefficients were sampled from a distribution with the specified standard deviation or standard error. For the PSA, 1000 bootstrap iterations of 1000 patients were simulated

$B M I$ body mass index, $H b A_{1 c}$ glycated hemoglobin, NPH neutral protamine Hagedorn, PSA probabilistic sensitivity analysis, $S M B G$ self-monitoring of blood glucose, UKPDS United Kingdom Prospective Diabetes Study

medications (aspirin, statins, and angiotensin-converting enzyme (ACE) inhibitors), screening for renal disease, retinopathy and diabetic foot complications, and post-complication management such as intensive insulin treatment after myocardial infarction. The cost of diabetes-related complications in the year of the event and the annual follow-up costs (applied in each year of the simulation subsequent to the event) were identified through a micro-costing approach. Studies describing the resource use associated with the treatment of diabetes-related complications in the Netherlands were identified, with Netherlands-specific unit costs applied to calculate annual costs. A summary of the costs of medicines and complications is provided in the Electronic supplementary material (ESM; Tables S1-S3).

\section{Utilities}

Utilities and disutilities associated with complications of diabetes were obtained from published sources [13, 18-21]. Quality-adjusted life expectancy was assessed using the additive CORE Default Method, which involves taking the lowest state utility associated with existing complications and adding event utilities for any events that occur in that year to create annual utility scores for each simulated patient [13].

\section{Sensitivity Analyses}

Sensitivity analyses were performed on key parameters in the model to assess the robustness of the base case findings (Table 3). These analyses varied model assumptions, or replaced a base case parameter with an alternative published data point.

\section{RESULTS}

\section{Base Case Analysis}

Projections of long-term clinical outcomes in patients previously uncontrolled on basal insulin indicated that IDegLira was associated with an improvement in quality-adjusted life expectancy of 0.43 QALYs versus IGlar $\mathrm{U} 100+3 \times$ IAsp treatment (Table 4). Clinical benefits resulted from a reduced incidence of diabetes-related complications in the IDegLira arm over the 50-year time horizon of the analysis. In addition to reducing the projected cumulative incidence of complications, IDegLira was associated with a delayed mean time to onset of diabetes-related complications. Mean time to onset of any diabetes-related complication in the modeling analysis was approximately 0.5 years longer with IDegLira than with IGlar $\mathrm{U} 100+3 \times$ IAsp, with benefits observed across all included micro- and macrovascular complications. 
Table 4 Base case analysis

\begin{tabular}{llll}
\hline Mean (SD) & IDegLira & IGlar U100 + 3 X IAsp & Difference \\
\hline Discounted life expectancy (years) & 16.74 & 16.49 & 0.26 \\
Discounted quality-adjusted life expectancy (QALYs) & 10.61 & 10.18 & 0.43 \\
Discounted direct costs (EUR) & 58,014 & 62,693 & -4679 \\
ICER (life expectancy) & IDegLira dominant & \\
ICER (quality-adjusted life expectancy) & IDegLira dominant & \\
\hline
\end{tabular}

$I C E R$ incremental cost-effectiveness ratio, $Q A L Y$ quality-adjusted life year, $S D$ standard deviation

Evaluation of the direct medical costs suggested that the mean cost per patient in the IDegLira arm was EUR 4679 less than in the IGlar U100 $+3 \times$ IAsp arm over patients' lifetimes (Table 4). The key driver of cost savings was the lower annual pharmacy cost with IDegLira compared with IGlar U100 + 3× IAsp over the first 5 years of the analysis. Further cost savings were identified as a result of avoiding diabetes-related complications, particularly cardiovascular complications, where cost savings of EUR 366 per patient were identified.

Quality-adjusted life expectancy was improved with IDegLira treatment at a cost saving from a healthcare payer perspective when compared with IGlar U100+3× IAsp. Therefore, IDegLira was considered dominant (improved clinical outcomes and lower costs) over IGlar U100 + 3× IAsp, and no calculation of an ICER was required (Table 4).

\section{One-way Sensitivity Analyses}

IDegLira remained dominant over IGlar $\mathrm{U} 100+3 \times$ IAsp in all sensitivity analyses (Table 5). Changes in most parameters resulted in only small changes in the calculated outcomes in the IDegLira arm (Table 5).

Changing the assumptions around treatment switching had a notable impact on the calculated health economic outcomes. Maintaining patients on IDegLira for longer increased the incremental clinical benefit and cost savings associated with IDegLira, whilst shortening the treatment duration had the converse effect. Applying an alternative $\mathrm{HbA}_{1 \mathrm{c}}$ progression with no increases applied at any stage of the analysis (attempting to replicate the legacy effect) resulted in an increased clinical benefit and increased cost savings in the IDegLira arm.

Applying the cost of NPH insulin (rather than IGlar U100) with no changes in dosing or clinical inputs resulted in reduced cost savings, as did discounting the pack price of IGlar U100 by $20 \%$. Using observed doses (i.e., not adjusted by the statistical model) to calculate annual treatment costs resulted in reduced cost savings with IDegLira, as did applying the maximum dose of IDegLira. Applying doses based on clinical practice in the Netherlands in the IGlar $\mathrm{U} 100+3 \times$ IAsp arm resulted in reduced cost savings with IDegLira, but IDegLira remained dominant.

Reducing the costs of SMBG test strips reduced the cost savings with IDegLira, but the conclusion of dominance did not change.

\section{Probabilistic Sensitivity Analysis}

The incremental cost-effectiveness scatterplot presents the incremental costs versus incremental effectiveness (QALYs gained) for IDegLira versus IGlar U100 + 3× IAsp (Fig. 1a), and shows 1000 mean values, each from a cohort of 1000 patients run through the model with sampling from distributions around model input parameters. The majority $(92.8 \%)$ of the points fell in the lower right quadrant, with both increased effectiveness (i.e., incremental quality-adjusted life expectancy) and reduced 
Table 5 Sensitivity analyses

\begin{tabular}{|c|c|c|c|}
\hline & $\begin{array}{l}\Delta \text { Cost } \\
\text { (EUR) }\end{array}$ & $\begin{array}{l}\Delta \text { Quality-adjusted } \\
\text { life expectancy } \\
\text { (QALYs) }\end{array}$ & $\begin{array}{l}\text { ICER } \\
\text { (EUR per } \\
\text { QALY gained) }\end{array}$ \\
\hline Base case & -4679 & +0.43 & Dominant \\
\hline 30-year time horizon (base case 50 years) & -4859 & +0.39 & Dominant \\
\hline Statistically significant treatment differences only & -4694 & +0.42 & Dominant \\
\hline $\mathrm{HbA}_{1 \mathrm{c}}$ benefit maintained & -5057 & +0.54 & Dominant \\
\hline UKPDS $\mathrm{HbA}_{1 \mathrm{c}}$ creep & -4794 & +0.40 & Dominant \\
\hline Upper $95 \% \mathrm{CI}$ of $\mathrm{HbA}_{1 \mathrm{c}}$ change in IDegLira arm & -5043 & +0.45 & Dominant \\
\hline Lower $95 \% \mathrm{CI}$ of $\mathrm{HbA}_{1 \mathrm{c}}$ change in IDegLira arm & -4873 & +0.41 & Dominant \\
\hline $\begin{array}{l}50 \% \text { of BMI benefit maintained after treatment switch } \\
\text { (base case BMI benefit abolished on switch) }\end{array}$ & -4681 & +0.53 & Dominant \\
\hline Treatment switch at 3 years in IDegLira arm (base case 5 years) & -3073 & +0.33 & Dominant \\
\hline Treatment switch at 7 years in IDegLira arm (base case 5 years) & -6454 & +0.52 & Dominant \\
\hline Treatment switch at 10 years in IDegLira arm (base case 5 years) & -8518 & +0.66 & Dominant \\
\hline NPH cost applied (base case IGlar U100 cost) & -3122 & +0.43 & Dominant \\
\hline $20 \%$ reduction in IGlar U100 price & -3967 & +0.43 & Dominant \\
\hline Observed doses (base case doses based on pooled analysis) & -3604 & +0.43 & Dominant \\
\hline Maximum dose of IDegLira (base case doses based on pooled analysis) & -2023 & +0.43 & Dominant \\
\hline Netherlands-based doses (base case doses based on pooled analysis) & -2588 & +0.43 & Dominant \\
\hline Lowest-price SMBG & -460 & +0.43 & Dominant \\
\hline Average-price SMBG & -3563 & +0.43 & Dominant \\
\hline Lowest-price needles & -4547 & +0.43 & Dominant \\
\hline Average-price needles & -4836 & +0.43 & Dominant \\
\hline Cost of complications $+20 \%$ & -4818 & +0.43 & Dominant \\
\hline Cost of complications $-20 \%$ & -4541 & +0.43 & Dominant \\
\hline Hypoglycemia utilities from Currie et al. [22] & -4679 & +0.41 & Dominant \\
\hline
\end{tabular}

$B M I$ body mass index, $C I$ confidence interval, ICER incremental cost-effectiveness ratio, NPH neutral protamine Hagedorn, $S M B G$ self-measured blood glucose

total costs for IDegLira compared with IGlar $\mathrm{U} 100+3 \times$ IAsp. A further $3.4 \%$ of points fell in the upper right quadrant, with increased effectiveness and increased costs.

The data from the scatterplot were used to generate a cost-effectiveness acceptability curve
(CEAC, Fig. 1b). Based on this analysis, assuming a willingness-to-pay threshold of EUR 20,000 per QALY gained, the modeling analysis indicated that there was a $99.6 \%$ probability that IDegLira is cost-effective versus IGlar U100 + 3× IAsp. 


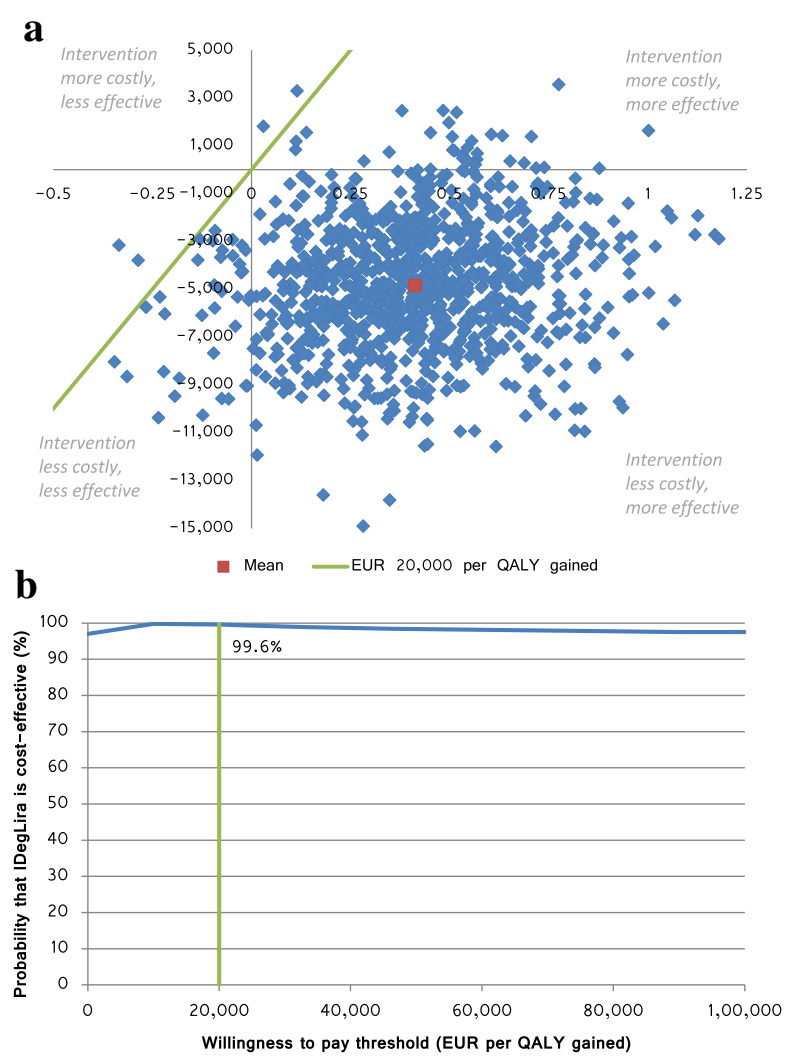

Fig. 1 a The incremental cost-effectiveness scatterplot presents the incremental costs versus the incremental effectiveness (QALYs gained) for IDegLira versus IGlar $\mathrm{U} 100+3 \times$ IAsp, and shows 1000 mean values, each of which is from a cohort of 1000 patients run through the model with sampling from distributions around model input parameters. b The data from the scatterplot were used to generate a cost-effectiveness acceptability curve (CEAC). The CEAC plots a range of cost-effectiveness thresholds on the horizontal axis against the probability that the intervention will be cost-effective at that threshold on the vertical axis

\section{DISCUSSION}

This long-term economic evaluation suggests that, from a healthcare payer perspective in the Netherlands, for patients with T2DM uncontrolled on basal insulin, IDegLira is likely to be dominant over IGlar $\mathrm{U} 100+3 \times$ IAsp (basal-bolus therapy), as it is less costly and more effective. In the base case analysis, IDegLira was associated with improved clinical outcomes, driven by multifactorial improvements in risk factors (such as $\mathrm{HbA}_{1 \mathrm{c}}$, systolic blood pressure, serum lipids, body mass index, and hypoglycemia) compared with IGlar $\mathrm{U} 100+3 \times$ IAsp. These favorable changes in physiological parameters resulted in benefits in both duration and quality of life.

The clinical benefits were achieved at a cost saving from a healthcare payer perspective. The key driver of this was the lower annual treatment costs with IDegLira compared with IGlar $\mathrm{U} 100+3 \times$ IAsp, with lower daily drug costs, needle costs, and SMBG costs. Based on the modeled doses and Netherlands-specific unit costs, IDegLira was associated with a pharmacy cost saving of EUR 1050 per patient per year. Further cost savings as a result of avoided diabetes-related complications were also identified in the IDegLira arm.

Extensive sensitivity analyses found that the conclusions were robust to changes in input parameters and modeling assumptions. IDegLira remained dominant over IGlar U100 $+3 \times$ IAsp in all scenarios investigated. Probabilistic sensitivity analysis showed a very high probability $(99.6 \%)$ that IDegLira would be cost-effective at a willingness-to-pay threshold of EUR 20,000 per QALY gained.

The present study was designed to capture the most appropriate comparator for patients failing to achieve glycemic control on basal insulin therapy in the Netherlands. Most commonly in the Netherlands, this is through the addition of fast-acting prandial insulin to basal insulin. IDegLira represents an alternative to basal-bolus therapy, and takes advantage of the complementary mechanisms of action of the two constituent agents to offer effective glycemic control without an elevated risk of hypoglycemia or weight gain. However, to date, no head-to-head trials comparing IDegLira with basal-bolus therapy have been published (results from an ongoing trial head-to-head trial, DUALVII, are expected later this year). In the absence of head-to-head data, a statistical indirect pooled analysis was performed, which could be considered a shortcoming of the present analysis. However, selection of the most appropriate comparator was the first priority in the analysis, and the application of evidence synthesis using robust methodologies is becoming increasingly important (and 
accepted) for health technology assessment globally [23]. The pooled analysis uses individual patient level data, which allows for a more robust analysis than aggregated study level data. The methodology is recognized by the European Network for Health Technology Assessment (EUNETHTA) guidelines on how to conduct indirect analyses [24], and has been used previously [25].

A potential limitation of the study, common to a number of health economic analyses, is the reliance on relatively short-term clinical trial data to make long-term projections. In terms of uncertainty around making long-term projections from short-term data, this remains one of the essential tenets of health economic modeling and it is still arguably one of the best of the available options to inform decision-making in the absence of long-term clinical trial data. Whilst there is always an element of clinical doubt around the accuracy of such an approach, every effort was made in the present analysis to minimize this, primarily by using a model of diabetes that has been extensively published and validated against real-life data both on first publication and recently, following a series of model updates $[12,26]$. It is recommended that outcomes should be projected over patient lifetimes in guidelines for the economic evaluation of interventions for patients with diabetes mellitus [27].

In order to increase the number of patients informing input values in the basal-bolus therapy arm, the pooled analysis used to inform this cost-effectiveness analysis included patients who previously received basal insulin and were randomly allocated to receive IGlar $\mathrm{U} 100+3 \times$ IAsp and IDeg $+3 \times$ IAsp [9]. Whilst pooled treatment effects were used to explore changes in physiological parameters, unit costs of IGlar U100 were used to calculate annual treatment costs (rather than weighting the costs by the proportion receiving IGlar U100 or IDeg in the trial). This is likely to be a conservative approach, since IDeg is associated with lower rates of hypoglycemia and reduced weight gain compared with IGlar U100 but IGlar U100 is associated with a lower cost.

Health economic guidance for the Netherlands recommends that a societal perspective, capturing both direct and indirect costs, should be used [14]. Due to a lack of country-specific data around days off work following each of the included diabetes-related complications, the present analysis took a healthcare payer perspective. It is likely that cost savings with IDegLira would be larger if indirect costs were included in the analysis, as IDegLira was associated with a reduced incidence and increased time to onset of diabetes-related complications. Therefore, the present analysis takes a conservative approach to capturing costs.

IDegLira is an effective treatment option for patients with T2DM uncontrolled on basal insulin, offering a reduced risk of hypoglycemia and weight gain versus basal-bolus therapy $[8,9]$, both of which are common barriers to treatment intensification [8]. IDegLira may also offer advantages from an adherence perspective, as it is associated with less nausea than typically observed with GLP-1 RAs, a likely result of the gradual increase in the dose of the liraglutide component of IDegLira during dose titration [28]. Furthermore, the once-daily dosing of IDegLira means that patients have a simple treatment option with reduced treatment complexity, with up to three fewer daily injections than basal-bolus regimens. The combination of IDeg and liraglutide in a single pen device means that patients will only need to perform a single dose adjustment, and resource use costs (e.g., needles and SMBG testing) will be lower than with basal-bolus therapy.

\section{CONCLUSIONS}

This study investigated the cost-utility of IDegLira versus IGlar U100 $+3 \times$ IAsp (basal-bolus therapy) in patients with T2DM uncontrolled on basal insulin from the healthcare payer perspective in the Netherlands. The results suggest that IDegLira is cost-effective in this setting, providing a simple basal insulin intensification option without increased risk of hypoglycemia or weight gain, and without an increased number of daily injections versus basal-bolus therapy. 


\section{ACKNOWLEDGEMENTS}

This study was funded by Novo Nordisk. Ossian Health Economics and Communications received consulting fees from Novo Nordisk to support the analysis. DRG Abacus received consulting fees from Novo Nordisk for medical writing and editorial support. Editorial assistance in the preparation of this manuscript was provided by Dr. Carrie Fidler of DRG Abacus. Support for this assistance was funded by Novo Nordisk. All named authors meet the International Committee of Medical Journal Editors (ICMJE) criteria for authorship of this manuscript, had full access to all data in this study, and take complete responsibility for the integrity of the data and accuracy of the data analysis. Barnaby Hunt performed the analysis and interpretation of results. Divina Glah, Barnaby Hunt, and Maarten van der Vliet contributed to the study design, data collection, and the interpretation of the results. All authors contributed to the writing, reviewing, and final approval of this manuscript.

Disclosures. Divina Glah is an employee of Novo Nordisk. Maarten van der Vliet is an employee of Novo Nordisk. Barnaby Hunt is an employee of Ossian Health Economics and Communications. Ossian received consulting fees from Novo Nordisk to support the analysis. DRG Abacus received consulting fees from Novo Nordisk to support the development of the manuscript.

Compliance with Ethics Guidelines. This article is based on previously conducted studies, and does not involve any new studies of human or animal subjects performed by any other authors.

Data Availability. Supplementary data available: Hunt_Supplementary Table S1-S3. All data generated or analyzed during this study are included in this published article as supplementary information files.

Open Access. This article is distributed under the terms of the Creative Commons Attribution-NonCommercial 4.0 International
License (http://creativecommons.org/licenses/ by-nc/4.0/), which permits any noncommercial use, distribution, and reproduction in any medium, provided you give appropriate credit to the original author(s) and the source, provide a link to the Creative Commons license, and indicate if changes were made.

\section{REFERENCES}

1. Campbell RK, Martin TM. The chronic burden of diabetes. Am J Manag Care. 2009;15(9 Suppl):S248-54.

2. International Diabetes Federation (IDF). Diabetes atlas. 7th ed (2015). http://www.idf.org/idf-diabetesatlas-seventh-edition. Accessed 5 May 2017

3. Rutten GEHM, De Grauw WJC, Nijpels G, Houweling ST, Van de Laar F, Bilo HJ, et al. NHG-standaard diabetes mellitus type 2 (derde herziening). Huisarts Wet. 2013;56(10):512-25.

4. InEen. Transparante ketenzorg diabetes mellitus, COPD en VRM rapportage zorggroepen over 2014. http://ineen.nl/wp-content/uploads/2015/06/1506 02-TRANSPARANTE-KETENZORG-2014.pdf. Accessed 5 May 2017

5. Overbeek JA, Houben E, Kring SI, Briers J, Penning-van Beest FJA, van der Heijden AAWA, et al. Intensification of basal insulin treatment among patients with diabetes mellitus type 2 in the Netherlands. Presented at the ISPOR 18th Annual European Congress; 2015 Nov 7-11; Milan, Italy. https://www.ispor.org/research_study_digest/details. asp. Accessed 5 May 2017

6. Bøgelund M, Vega-Hernandez G, Lopes S, Schaper N. Barriers to intensification of insulin treatment in patients with type 2 diabetes in the Netherlands: assessing patient preferences and behaviours. Value Health. 2015;18:A612.

7. Buse JB, Vilsboll T, Thurman J, Blevins TC, Langbakke IH, Bottcher SG, et al. Contribution of liraglutide in the fixed-ratio combination of insulin degludec and liraglutide (IDegLira). Diabetes Care. 2014;37(11):2926-33.

8. Lingvay I, Manghi FP, Garcia-Hernandez P, Norwood P, Lehmann L, Tarp-Johansen MJ, et al. Effect of insulin glargine up-titration vs insulin degludec/ liraglutide on glycated hemoglobin levels in patients with uncontrolled type 2 diabetes: the DUAL $\mathrm{V}$ randomized clinical trial. JAMA. 2016;315(9):898-907. 
9. Freemantle N, Mamdani M, Vilsboll T, Kongso JH, Kvist K, Bain SC. IDegLira versus alternative intensification strategies in patients with type 2 diabetes inadequately controlled on basal insulin therapy. Diabetes Ther Res Treat Educ Diabetes Relat Disord. 2015;6(4):573-91.

10. Sassi F. Calculating QALYs, comparing QALY and DALY calculations. Health Policy Plan. 2006;21(5): 402-8.

11. Boersma C, Broere A, Postma MJ. Quantification of the potential impact of cost-effectiveness thresholds on Dutch drug expenditures using retrospective analysis. Value Health. 2010;13(6):853-6.

12. McEwan P, Foos V, Palmer JL, Lamotte M, Lloyd A, Grant D. Validation of the IMS CORE diabetes model. Value Health. 2014;17(6):714-24.

13. Palmer AJ, Roze S, Valentine WJ, Minshall ME, Foos $\mathrm{V}$, Lurati FM, et al. The CORE diabetes model: projecting long-term clinical outcomes, costs and cost-effectiveness of interventions in diabetes mellitus (types 1 and 2) to support clinical and reimbursement decision-making. Curr Med Res Opin. 2004;20(Suppl 1):S5-26.

14. College voor zorgverzekeringen. Guidelines for pharmacoeconomic research, updated version (2006). http://www.ispor.org/PEguidelines/source/ HTAGuidelinesNLupdated2006.pdf. Accessed 5 May 2017

15. World Health Organization (WHO) World health statistics 2012. Life tables for WHO member states. http://www.who.int/whosis/database/life_tables/ life_tables.cfm. Accessed 5 May 2017

16. Eriksen M, Mackay J, Ross H. The tobacco atlas. 4th ed. http://www.tobaccoatlas.org/uploads/Images/ PDFs/Tobacco_Atlas_2ndPrint.pdf. Accessed 5 May 2017

17. World Health Organization (WHO). Global status report on alcohol and health 2014. http://apps. who.int/iris/bitstream/10665/112736/1/978924069 2763_eng.pdf. Accessed 5 May 2017

18. Bagust A, Beale S. Modelling EuroQol health-related utility values for diabetic complications from CODE-2 data. Health Econ. 2005;14(3):217-30.

19. Clarke P, Gray A, Holman R. Estimating utility values for health states of type 2 diabetic patients using the EQ-5D (UKPDS 62). Med Decis Making. 2002;22(4):340-9.
20. Evans M, Khunti K, Mamdani M, Galbo-Jorgensen CB, Gundgaard J, Bogelund M, et al. Health-related quality of life associated with daytime and nocturnal hypoglycaemic events: a time trade-off survey in five countries. Health Qual Life Outcomes. 2013;11(1):90.

21. Tengs TO, Wallace A. One thousand health-related quality-of-life estimates. Med Care. 2000;38(6): 583-637.

22. Currie CJ, Poole CD, Woehl A, Morgan CL, Cawley $\mathrm{S}$, Rousculp MD, et al. The health-related utility and health-related quality of life of hospital-treated subjects with type 1 or type 2 diabetes with particular reference to differing severity of peripheral neuropathy. Diabetologia. 2006;49(10):2272-80.

23. Dias SW, Sutton NJ, Sutton AJ, Ades AE. NICE DSU technical support document 1: introduction to evidence synthesis for decision making, 2011 (last updated April 2012). http://scharr.dept.shef.ac.uk/ nicedsu/technical-support-documents/evidencesynthesis-tsd-series/. Accessed 5 May 2017

24. EUNETHTA. Guideline-comparators \& comparisons: direct and indirect comparisons. February 2013. https://5026.fedimbo.belgium.be/sites/5026. fedimbo.belgium.be/files/Direct\%20and\%20indirect \%20comparisons.pdf. Accessed 5 May 2017

25. Zinman B, Schmidt WE, Moses A, Lund N, Gough S. Achieving a clinically relevant composite outcome of an $\mathrm{HbA1c}$ of $<7 \%$ without weight gain or hypoglycaemia in type 2 diabetes: a meta-analysis of the liraglutide clinical trial programme. Diabetes Obes Metab. 2012;14(1):77-82.

26. Palmer AJ, Roze S, Valentine WJ, Minshall ME, Foos $\mathrm{V}$, Lurati FM, et al. Validation of the CORE diabetes model against epidemiological and clinical studies. Curr Med Res Opin. 2004;20(Suppl 1):S27-40.

27. American Diabetes Association Consensus Panel. Guidelines for computer modeling of diabetes and its complications. Diabetes Care. 2004;27(9): 2262-5.

28. Aroda V, Jaeckel E, Jarlov H, Abrahamsen T, Vilsbøll $\mathrm{T}$. Incidence of gastrointestinal side effects similar between IDegLira and non-GLP-1 RA comparators. Presented at ADA 2015; 2015 June 5-9; Boston, MA, USA. 